OPEN ACCESS

Edited by:

Hinsby Cadillo-Quiroz,

Arizona State University,

United States

Reviewed by:

Heli Juottonen,

University of Jyväskylä, Finland

Carl-Eric Wegner,

Friedrich Schiller University Jena,

Germany

*Correspondence:

Hongmei Wang

hmwang@cug.edu.cn;

wanghmei04@163.com

Specialty section:

This article was submitted to Terrestrial Microbiology,

a section of the journal

Frontiers in Microbiology

Received: 31 March 2019

Accepted: 04 July 2019

Published: 24 July 2019

Citation:

Tian W, Wang $H$, Xiang $X$, Wang $R$ and Xu Y (2019) Structural Variations

of Bacterial Community Driven by

Sphagnum Microhabitat

Differentiation in a

Subalpine Peatland.

Front. Microbiol. 10:1661.

doi: 10.3389/fmicb.2019.01661

\section{Structural Variations of Bacterial Community Driven by Sphagnum Microhabitat Differentiation in a Subalpine Peatland}

\author{
Wen Tian', Hongmei Wang ${ }^{1,2 \star}$, Xing Xiang ${ }^{1}$, Ruicheng Wang ${ }^{1}$ and $Y_{i n g} X^{1}$ \\ 'State Key Laboratory of Biogeology and Environmental Geology, China University of Geosciences, Wuhan, China, \\ 2Laboratory of Basin Hydrology and Wetland Eco-Restoration, China University of Geosciences, Wuhan, China
}

Sphagnum microbiomes play an important role in the northern peatland ecosystems. However, information about above and belowground microbiomes related to Sphagnum at subtropical area remains largely limited. In this study, microbial communities from Sphagnum palustre peat, S. palustre green part, and S. palustre brown part at the Dajiuhu Peatland, in central China were investigated via 16S rRNA gene amplicon sequencing. Results indicated that Alphaproteobacteria was the dominant class in all samples, and the classes Acidobacteria and Gammaproteobacteria were abundant in S. palustre peat and S. palustre brown part samples, respectively. In contrast, the class Cyanobacteria dominated in S. palustre green part samples. Microhabitat differentiation mainly contributes to structural differences of bacterial microbiome. In the $S$. palustre peat, microbial communities were significantly shaped by water table and total nitrogen content. Our study is a systematical investigation on above and belowground bacterial microbiome in a subalpine Sphagnum peatland and the results offer new knowledge about the distribution of bacterial microbiome associated with different microhabitats in subtropical area.

Keywords: subalpine peatland, S. palustre, microhabitat, microbial diversity, water table, total nitrogen

\section{INTRODUCTION}

Peatlands only cover approximately $3 \%$ of the Earth's land surface, but store up to $\sim 30 \%$ of the terrestrial carbon and thus play a critical role in global carbon cycling (Yu et al., 2010; Mitsch et al., 2013). Sphagnum-dominated peatlands are widely distributed in the northern hemisphere (Shaw et al., 2010; Jassey et al., 2011; Weston et al., 2015). Sphagnum mosses maintain their dominance through high competition for cation exchange (Soudzilovskaia et al., 2010) and inhibition of growth of other plants attributed to $\mathrm{H}^{+}$release (Weston et al., 2015; Kostka et al., 2016). Due to the dominance and widespread occurrence of Sphagnum, the growth of mosses is directly linked to the ecological function of peatland ecosystems.

As the phylogenetically oldest land plant on the Earth, rootless mosses interact with microorganisms (Opelt et al., 2007a) and form highly specific microbiomes (Opelt et al., 2007b; Bragina et al., 2012b, 2015). Recently, Sphagnum-associated microbiomes have been demonstrated to greatly affect the growth and health of host mosses (Weston et al., 2015; Kostka et al., 2016). For example, symbiotic methanotrophs and diazotrophs in Sphagnum have been confirmed to significantly 
contribute to $\mathrm{CH}_{4}$ consumption in peatlands, $\mathrm{CO}_{2}$ and nitrogen supply for Sphagnum growth (Kip et al., 2010, 2012; Bragina et al., 2012a, 2013; Berg et al., 2013). Moreover, microbial methane oxidation potential differs among different parts of submerged and non-submerged Sphagnum (Raghoebarsing et al., 2005), which may suggest the difference of microbial communities in different parts of Sphagnum. Indeed, bacterial 16S rRNA gene copy numbers and community compositions vary between the green part (living part doing the photosynthesis) and the brown part (dying part submerging into the water) of Sphagnum (Xiang et al., 2014). These results have stimulated a series of investigation about Sphagnum microbiomes (Bragina et al., 2014, 2015) and have expanded our knowledge about the interactions between mosses and microbiomes in peatland ecosystems.

Belowground microbial communities, the drivers of elemental cycling in peatland ecosystems, have also been investigated across different climatic zones, such as high-latitude regions (Belova et al., 2006; Kulichevskaia et al., 2006; Pankratov et al., 2008), tropical rainforest swamps and peatlands (Jackson et al., 2009; Kanokratana et al., 2011; Mishra et al., 2014). A common finding is that microbial communities in peatland ecosystems are mainly composed of several phyla such as Acidobacteria and Proteobacteria (Gilbert and Mitchell, 2006; Andersen et al., 2013). Despite the common dominant taxa in different peatlands, the correlations between microbial communities and environmental factors vary. For instance, water table is demonstrated to influence on the structure of microbial communities (Jaatinen et al., 2007; Kwon et al., 2013; Mishra et al., 2014) or their alpha diversity (Chambers et al., 2016; Urbanová and Barta, 2016; Zhong et al., 2017) in peatlands. $\mathrm{pH}$ has been reported to affect soil bacterial communities globally (Bahram et al., 2018) as well as peat microbial communities (Hartman et al., 2008; Lin et al., 2012; Urbanová and Bárta, 2014). Other environmental factors such as temperature and nitrogen content (Pankratov et al., 2008), organic matter content, moisture, and phosphorus (Elliott et al., 2015) also show their impact on microbial communities in peatland ecosystems.

Due to the limited distribution and accessibility of peatlands in subtropical areas, microbiomes remain enigmatic particularly those related to different microhabitats, i.e., different parts of Sphagnum. We hypothesize that (1) peatland bacterial microbiome in subtropical region may share the common dominant taxa with other peatland ecosystems but vary among different microhabitats; (2) water table may be one of the most important factors shaping bacterial microbiome in Sphagnum peat samples.

To test our hypothesis, the structures of microbial communities from S. palustre peat, S. palustre brown part, and S. palustre green part were investigated via $16 \mathrm{~S}$ rRNA gene Illumina sequencing and the relationships between bacterial communities and environmental factors were taken into account. The aim of this study was to investigate bacterial communities, especially the above and belowground Sphagnum-associated communities in a subtropical peatland. The specific goals were to examine (1) the structure (diversity and composition) of bacterial communities in different microhabitats, and (2) the relationship between peat bacterial communities and environmental factors in the Dajiuhu Peatland.

\section{MATERIALS AND METHODS}

\section{Study Area and Sampling}

The Dajiuhu Peatland $\left(31^{\circ} 24^{\prime} \sim 31^{\circ} 33^{\prime} \mathrm{N}, 109^{\circ} 56^{\prime} \sim 110^{\circ} 11^{\prime} \mathrm{E}\right)$, located in the Shennongjia Forestry District, Hubei province, central China (Figure 1A), is a typical subalpine peatland with a surface area of about $16 \mathrm{~km}^{2}$ and $1,730 \mathrm{~m}$ a.s.l. The mean annual precipitation and temperature are $1,560 \mathrm{~mm}$ and $7.2^{\circ} \mathrm{C}$, respectively (Huang et al., 2013). Organic matter is abundant due to the low temperature and high water table (Xiang et al., 2017). Vegetation in the peatland is mainly characterized by Carex argyi, C. capillacea, S. palustre, Sanguisorba officinalis, and Euphorbia esula with shrubs grown on patches of dry land, which divide the peatland into several small sections. The details of vegetation patterns and hydrological characteristics about the study area have been described previously (Huang et al., 2012; Li et al., 2016).

Samples were collected from four sites in the core area of the Dajiuhu Peatland, which are Erhaoba (EHB), Niangniangfen (NNF), and two sites at Yangluchang (YLC) with different water table (Figure 1B) in July 2016. Water table is expressed with negative number when it is below the peat surface and vice versa. At each site, $S$. palustre peat $(0-5 \mathrm{~cm})$ and $S$. palustre were collected in triplicates. Underlying $S$. palustre peat samples were stored in 50-ml sterile centrifuge tubes and $S$. palustre samples were placed in sterile plastic bags. All samples were transported to the geomicrobiology laboratory at China University of Geosciences (Wuhan) on dry ice within $12 \mathrm{~h}$. S. palustre brown part and $S$. palustre green part were cut off aseptically according the color identification in the laboratory. In total, 36 samples [3 replicates $\times 3$ microhabitats $(S$. palustre peat, $S$. palustre brown part, and $S$. palustre green part $) \times 4$ sites] were examined. Visible plant roots, litter, and debris were removed from peat samples. Half of each peat sample was stored at $4^{\circ} \mathrm{C}$ for chemical analysis and the other half was stored at $-20^{\circ} \mathrm{C}$ for DNA extraction.

\section{Physicochemical Analysis}

S. palustre peat samples (approx. $20 \mathrm{~g}$ ) were dried at $50^{\circ} \mathrm{C}$ for $24 \mathrm{~h}$ to measure the water content. One half of each dried peat sample was ground to 200-mesh after fumigation with concentrated $\mathrm{HCl}$ (Horwáth and Kessel, 2001), and analyzed for total organic carbon (TOC) and total nitrogen (TN) with a Vario EL III Elemental Analyzer (Elementar, Germany). The other half was converted to ash at $550^{\circ} \mathrm{C}$ for $3 \mathrm{~h}$ to measure the organic matter content. Peat samples weighing $5 \mathrm{~g}$ were sonicated in deionized water ( $1: 4 \mathrm{w} / \mathrm{v}$ ratio) for $15 \mathrm{~min}$, followed by $20 \mathrm{~min}$ of shaking at $200 \mathrm{rpm}$. The suspension was centrifuged at 5,000 $\mathrm{g}$ for $5 \mathrm{~min}$ (Elliott et al., 2015) and the $\mathrm{pH}$ of the supernatant was measured with a multi-parameter water quality detector ( $\mathrm{HACH}$, Loveland, $\mathrm{CO})$. The supernatant was filtered 


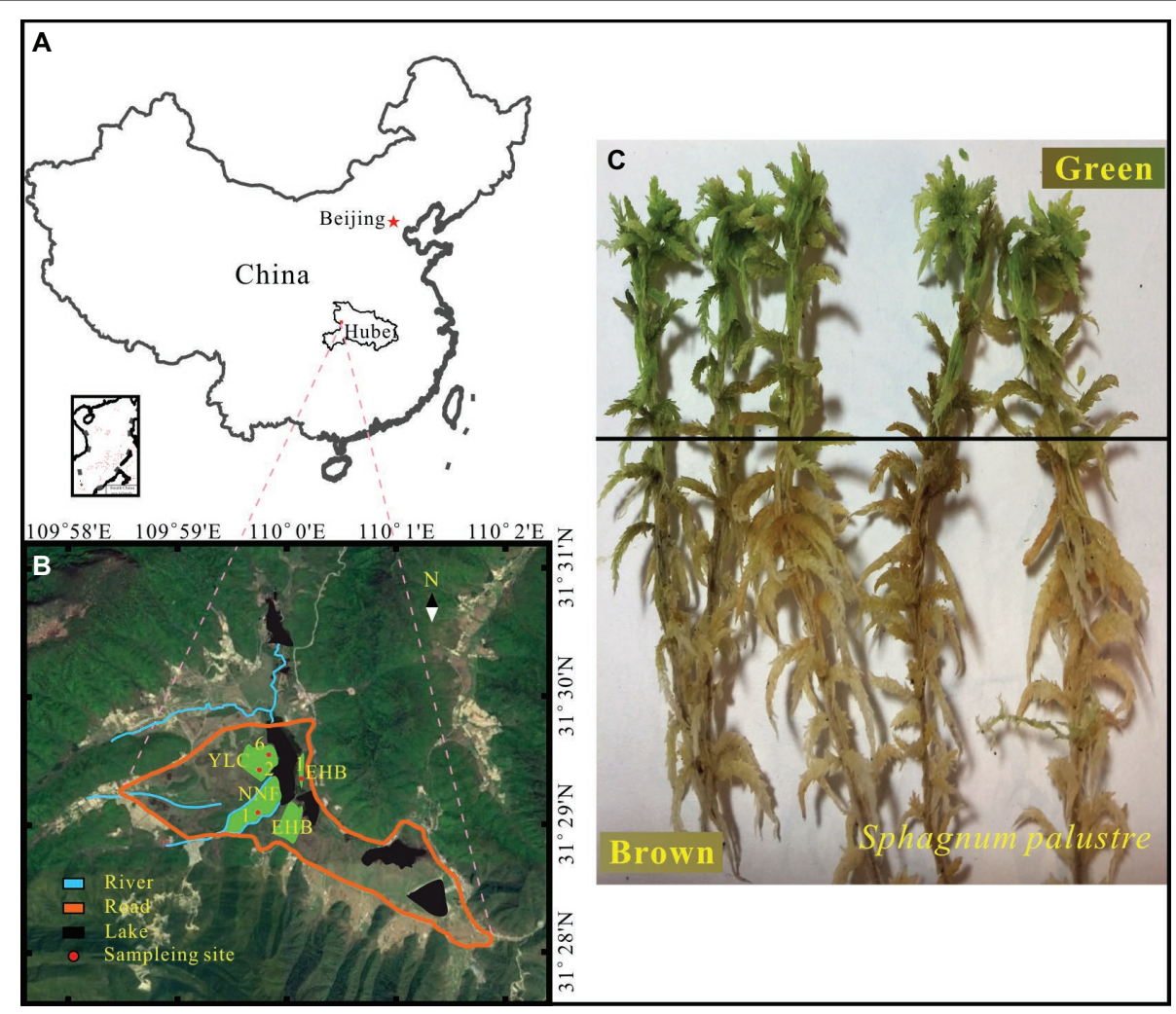

FIGURE 1 | Location of study area (A) and sampling sites (B) in the Dajiuhu Peatland, Hubei province, central China. (C) S. palustre brown part and S. palustre green part.

through $0.22-\mu \mathrm{m}$ membrane and the filtrate was analyzed for major anions and cations using an ICS-600 ion chromatograph (Yun et al., 2016).

\section{DNA Extraction, PCR, and Illumina Sequencing}

S. palustre samples were cut into two parts (Figure 1C) according to color identification and were designated as SB for S. palustre brown part and SG for $S$. palustre green part, respectively. The samples were rinsed three times with sterile distilled water for $5 \mathrm{~min}$ each time to remove adhesive debris before DNA extraction. Approximately $0.5 \mathrm{~g}$ of frozen-dried samples were subjected to DNA extraction with the PowerSoil DNA Isolation Kit (QIAGEN, Düsseldorf, NW) following the manufacturer's instruction (Xiang et al., 2014). Total DNA was quantified using a NanoDrop 2000 spectrophotometer (Thermo Fisher Scientific, Waltham, MA) and $1 \%$ agarose gel was used to check the DNA quality. The V3 and V4 regions of microbial $16 \mathrm{~S}$ rRNA were amplified using the primers $347 \mathrm{~F}$ (5'-CCTACGGRRBGCASCAGKVRVGAAT-3') and 802R (5'-GGACTACNVGGGTWTCTAATCC-3') (Cai et al., 2019). After amplification, PCR products were purified and quantified by Qubit 2.0 Fluorometer (Invitrogen, Carlsbad, CA). DNA libraries were validated by Agilent 2100 Bioanalyzer (Agilent Technologies, Palo Alto, CA), and then applied to
Illumina MiSeq sequencing with $2 \times 300$ bp paired-end at GENEWIZ, Inc. (Suzhou, China).

\section{Data Processing}

The raw sequences obtained from MiSeq reads were demultiplexed through the barcode sequences and processed with Quantitative Insight Into Microbial Ecology (QIIME 1.9.1 version) pipeline (Caporaso et al., 2010). The sequences of an individual sample were identified based on their unique barcode, which subsequently went through the removal of primers and barcodes, and paired-end assembly. The high-quality sequences were determined based on (1) absence of any ambiguous base "N", (2) length $>200 \mathrm{bp}$, and (3) base quality score $>20$ in a sliding window. The chloroplast was removed and the chimeric sequences were identified using the UCHIME algorithm (Edgar et al., 2011). The clean sequences were classified into operational taxonomic units (OTUs) by UCLUST (Edgar, 2010) based on 97\% sequence similarity and singletons (OTUs with only one sequence in all samples) were filtered out. Taxonomic category assignment was conducted with representative sequences at a threshold of 0.8 in the Ribosomal Database Program classifier (Cole et al., 2009), followed by annotation with SILVA 119 database. All the samples were resampled to the same number of reads $(29,549)$ using mothur (Schloss et al., 2009) prior to statistical analysis. 
The original 16S rRNA sequence data have been deposited in the NCBI Sequence Read Archive ${ }^{1}$ under the accession number PRJNA512496.

\section{Statistical Analysis}

One-way analysis of variance (ANOVA) was used to test the microhabitat (S. palustre peat, S. palustre brown part, and $S$. palustre green part) impact on alpha diversity and the relative abundance of different taxa in SPSS 18.0. Principal coordinate analysis (PCoA) based on Bray-Curtis distance metrics was conducted to visualize the community dissimilarity among the samples and recognize key factors driving variations of community structure. Permutational multivariate analysis of variance (PERMANOVA) with the Bray-Curtis and Jaccard distances matrixes was used to compare the dissimilarity degree of community among groups. PCoA coupled with PERMANOVA quantified the differences of $S$. palustre peat communities based on Bray-Curtis distances. Beta diversity analysis was performed using "vegan" package in $\mathrm{R}$ (v. 3.4.1). The indicator species in different microhabitats were identified via linear discriminant analysis effect size (LEfSe) (Segata et al., 2011). Regression model between water table and alpha diversity in $S$. palustre peat was performed in $\mathrm{R}$ (v. 3.4.1) "basicTrendline" package. The correlations between environmental factors and bacterial communities were revealed by redundancy analysis (RDA) using Canoco 5 (Braak and Šmilauer, 2015).

${ }^{1}$ https://www.ncbi.nlm.nih.gov/sra

\section{RESULTS}

\section{Geochemistry of S. palustre Peat}

The geochemical properties of $S$. palustre peat samples are listed in Supplementary Table S1. All peat samples were acidic, with $\mathrm{pH}$ ranging from 5.35 to 6.70 , and significant differences were observed $(\alpha=0.05)$ among the four sampling sites (Figure 2). Overall, the moisture and organic matter content (OM) were high (69.16-89.95\% and 60.87-97.72\%, respectively), and total nitrogen $(\mathrm{TN})$ was low (1.03-2.17\%) in all samples. Concentrations of $\mathrm{NO}_{3}^{-}, \mathrm{Na}^{+}$, and $\mathrm{Ca}^{2+}$ were significantly higher in samples at the first site of Erhaoba (EHB1) (mean $79.29 \mathrm{mg} \mathrm{kg}^{-1} \mathrm{NO}_{3}^{-}, 39.26 \mathrm{mg} \mathrm{kg}^{-1} \mathrm{Na}^{+}$, and $148.84 \mathrm{mg} \mathrm{kg}$ $\mathrm{Ca}^{2+}$ ) than those of samples at the first site of Niangniangfen (NNF1) (mean $16.56 \mathrm{mg} \mathrm{kg}^{-1} \mathrm{NO}_{3}^{-}, 13.59 \mathrm{mg} \mathrm{kg}^{-1} \mathrm{Na}^{+}$, and $\left.15.60 \mathrm{mg} \mathrm{kg}^{-1} \mathrm{Ca}^{2+}\right)$. Temperature $\left(23.1-30.3^{\circ} \mathrm{C}\right)$ and water table $(-8$ to $0 \mathrm{~cm})$ varied among $S$. palustre peat samples.

\section{Alpha and Beta Diversities of Bacterial Microbiome}

In total, 2,849 OTUs were recovered after quality control and resampled (archaea and unclassified excluded) (Supplementary Table S2). Alpha diversity was the highest in S. palustre peat samples, followed by $S$. palustre brown part. The $S$. palustre green part showed the lowest alpha diversity as indicated by OTU richness, ACE index, and Shannon index (Figure 3A). Shannon diversity of bacterial communities was statistically different between $S$. palustre peat and $S$. palustre $(\alpha=0.05)$, and between $S$. palustre brown part and $S$. palustre green part $(\alpha=0.05)$.

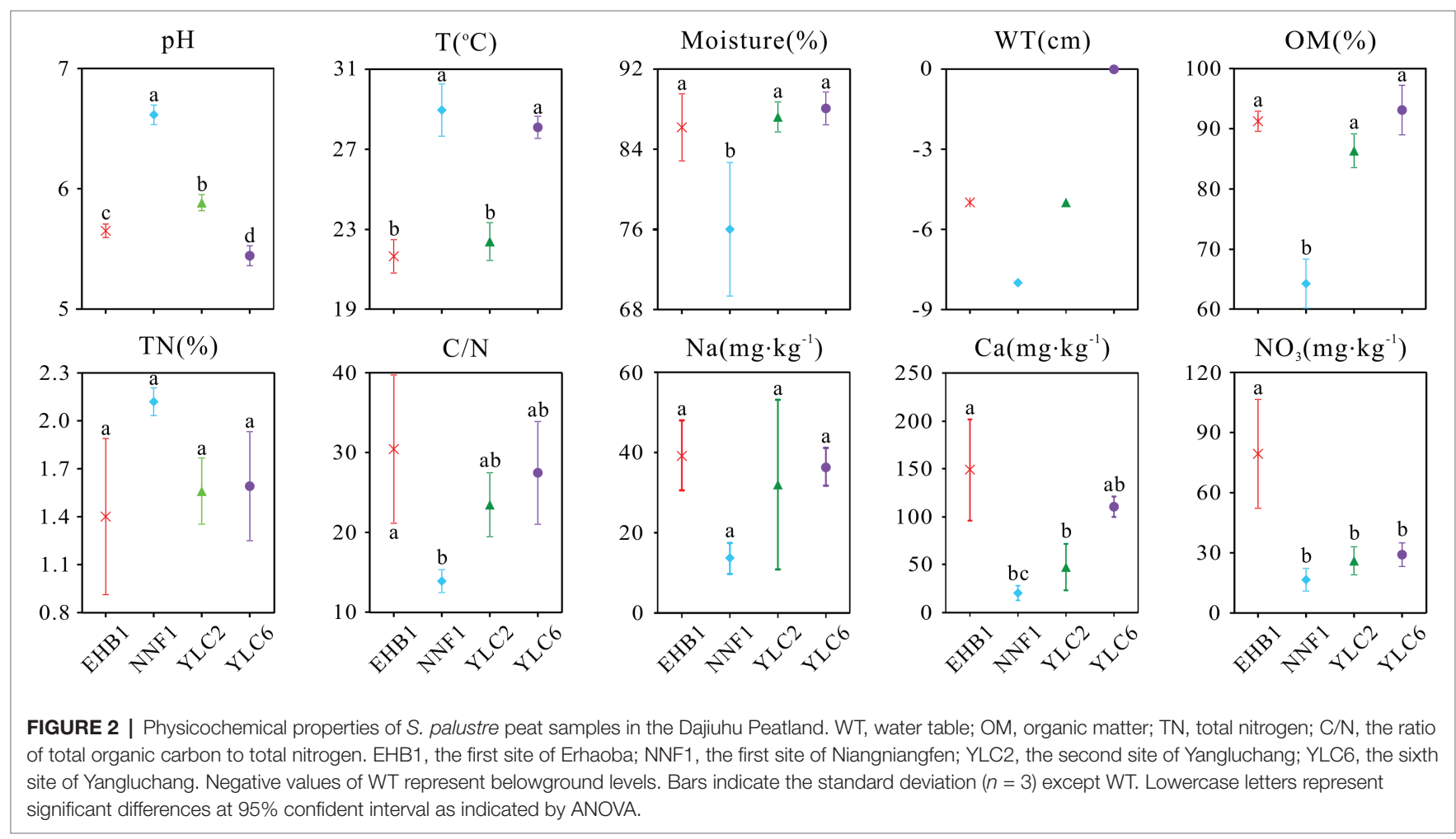


PCoA revealed a clear clustering pattern of bacterial communities at the OTU level according to different microhabitats of S. palustre. PCoA1 and PCoA2 in Bray-Curtis matrix explained $34.91 \%$ and $17.50 \%$ of the variance, respectively (Figure 3B). Bacterial communities between any two microhabitats were significantly $(\alpha=0.05)$ different as indicated by PERMANOVA both at the OTU and phylum levels. However, fewer differences were observed among sampling sites (Table 1).

\section{Taxonomic Composition of Bacterial Microbiome}

In total, 30 bacterial phyla and 41 classes were identified across all samples, and dominant taxa varied among different microhabitats (Figure 3C). In S. palustre peat samples, the majority of reads were assigned to Acidobacteria and Alphaproteobacteria. While in S. palustre brown part samples,
Alphaproteobacteria and Gammaproteobacteria were the dominant taxa. In S. palustre green part, Cyanobacteria and Alphaproteobacteria dominated the microbial communities. The relative abundance of Alphaproteobacteria and Cyanobacteria decreased from SG (24.21 \pm 10.28 and $47.36 \pm 21.05 \%)$ to $\mathrm{SB}(23.14 \pm 6.82$ and $9.61 \pm 6.98 \%)$ and further to SP $(20.79 \pm 6.00$ and $0.49 \pm 0.22 \%)$ (Supplementary Table S3). In contrast, Acidobacteria, Betaproteobacteria, and Deltaproteobacteria showed the opposite trend, which significantly increased from SG $(5.45 \pm 2.35,2.68 \pm 1.27 \%$ and $0.62 \pm 0.40 \%)$ to $\mathrm{SB}$ $(16.20 \pm 7.59,4.32 \pm 1.85$ and $2.57 \pm 1.89 \%)$ and further to $\operatorname{SP}(25.62 \pm 6.76,6.08 \pm 3.74$ and $6.17 \pm 2.84 \%)$. Acidobacteria and Deltaproteobacteria (ANOVA, $p<0.05$ ) were significantly enriched in $S$. palustre peat samples. While in $S$. palustre green part samples, Cyanobacteria (ANOVA, $p<0.05$ ) was
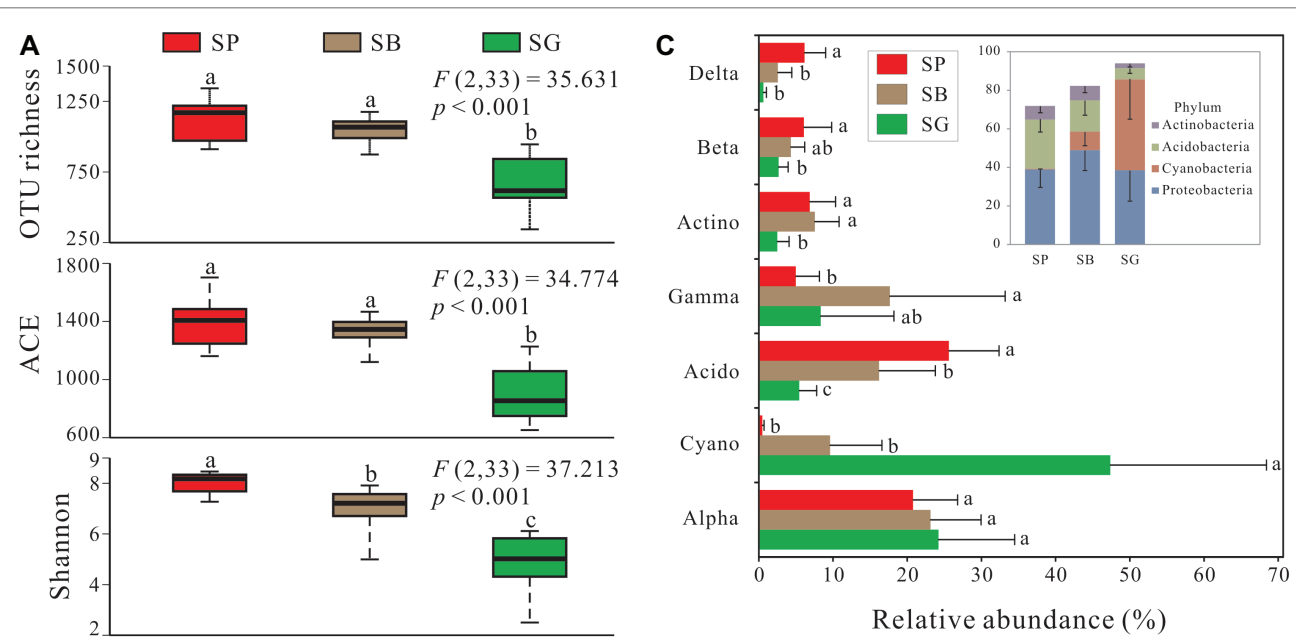

B

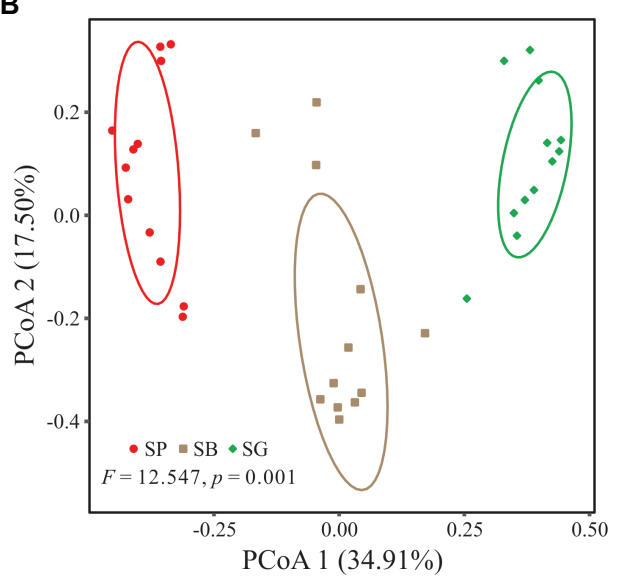

D

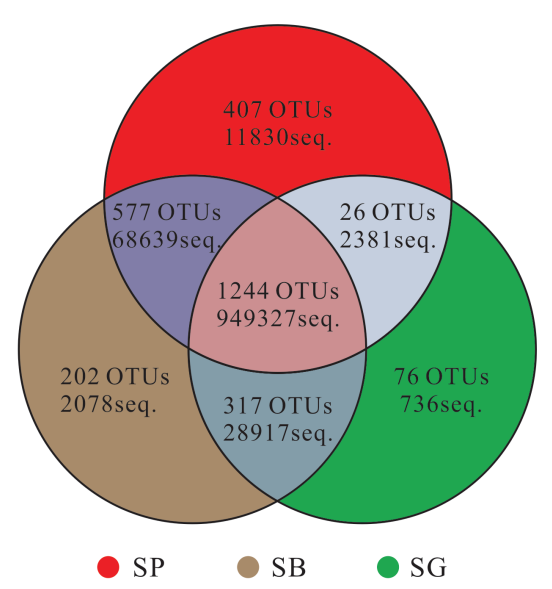

FIGURE 3 | Sphagnum-associated bacterial communities. (A) Alpha diversity of 16S rRNA genes in different microhabitats. Box plots show the maximum and minimum, median, first (25\%) and third (75\%) quartiles observed values in each dataset $(n=12)$. Data were analyzed by the one-way ANOVA with Tukey's HSD post hoc comparisons. The test statistical values of $\mathrm{F}(\mathrm{DFn}, \mathrm{DFd})$ are shown at the right top of each graph. Significant differences $(p<0.05)$ across groups are indicted with lowercase letters. (B) The principal coordinate analysis (PCOA) plots based on the weighted Bray-Curtis metrics among samples. Ellipses indicate 95\% confidence level. (C) Dominant phyla (relative abundance $>5 \%$ ) and corresponding classes' distribution in different microhabitats. (D) Numbers of mutual OTUs and unique OTUs in each microhabitat. Unclassified and archaea OTUs are removed. Acido, Acidobacteria; Actino, Actinobacteria; Alpha, Alphaproteobacteria; Beta, Betaproteobacteria; Cyano, Cyanobacteria; Delta, Deltaproteobacteria; Gamma, Gammaproteobacteria. SP, S. palustre peat; SB, S. palustre brown part; SG, S. palustre green part. 
TABLE 1 | PERMANOVA indicating dissimilarities of bacterial community among different microhabitats and sampling sites.

\begin{tabular}{|c|c|c|c|c|c|c|c|c|}
\hline \multirow{3}{*}{$\begin{array}{l}\text { Distance } \\
\text { Phylogenetic level } \\
\text { PERMANOVA output }\end{array}$} & \multicolumn{4}{|c|}{ Bray-Curtis } & \multicolumn{4}{|c|}{ Jaccard } \\
\hline & \multicolumn{2}{|c|}{ OTU } & \multicolumn{2}{|c|}{ Phylum } & \multicolumn{2}{|c|}{ OTU } & \multicolumn{2}{|c|}{ Phylum } \\
\hline & $\boldsymbol{F}$ & $p$ & $\boldsymbol{F}$ & $p$ & $\boldsymbol{F}$ & $p$ & $\boldsymbol{F}$ & $p$ \\
\hline \multicolumn{9}{|l|}{ Microhabitat } \\
\hline SP vs. SB & 7.56 & $0.003^{\star *}$ & 7.738 & $0.003^{\star \star}$ & 4.980 & $0.003^{\star \star}$ & 5.964 & $0.003^{\star \star}$ \\
\hline SP vs. SG & 21.65 & $0.003^{\star *}$ & 32.163 & $0.003^{\star \star}$ & 11.813 & $0.003^{\star *}$ & 21.248 & $0.003^{\star \star}$ \\
\hline SB vs. SG & 9.988 & $0.003^{\star *}$ & 21.511 & $0.003^{\star \star}$ & 6.572 & $0.003^{\star \star}$ & 14.184 & $0.003^{\star \star}$ \\
\hline \multicolumn{9}{|l|}{ Sampling site } \\
\hline EHB1 vs. NNF1 & 1.006 & 1.000 & 0.018 & 1.000 & 1.151 & 1.000 & 0.171 & 1.000 \\
\hline EHB1 vs. YLC2 & 0.954 & 1.000 & 2.045 & 0.798 & 1.067 & 1.000 & 1.912 & 0.696 \\
\hline EHB1 vs. YLC6 & 3.077 & 0.126 & 2.778 & 0.390 & 2.489 & 0.114 & 2.530 & 0.312 \\
\hline NNF1 vs. YLC2 & 1.269 & 1.000 & 3.020 & 0.408 & 1.344 & 1.000 & 2.614 & 0.378 \\
\hline NNF1 vs. YLC6 & 3.109 & 0.108 & 2.994 & 0.372 & 2.671 & $0.048^{\star}$ & 2.961 & 0.222 \\
\hline YLC2 vs. YLC6 & 3.081 & $0.036^{\star}$ & 4.967 & $0.042^{*}$ & 2.497 & 0.066 & 4.474 & $0.018^{*}$ \\
\hline
\end{tabular}

PERMANOVA, permutational multivariate analysis of variance; SP, S. palustre peat; SB, S. palustre brown part; SG, S. palustre green part; EHB1, the first site of Erhaoba; NNF1, the first site of Niangniangfen; YLC2, the second site of Yangluchang; YLC6, the sixth site of Yangluchang. F, test statistic; p's are corrected by false discovery rate (FDR). ${ }^{*} p \leq 0.05,{ }^{* *} p \leq 0.01$

significantly different compared to that in $S$. palustre peat and S. palustre brown part.

\section{Indicator Groups for Different Microhabitats}

The overview of OTU distribution among different microhabitats showed that 1,244 OTUs were shared by three microhabitats (Figure 3D). S. palustre peat and S. palustre brown part shared the most OTUs $(1,821)$, followed by that in $S$. palustre brown and green parts $(1,561)$. The least OTUs $(1,270)$ were shared between S. palustre peat and S. palustre green part. About 407 of the total OTUs were exclusively observed in S. palustre peat as compared to $S$. palustre brown part (202 OTUs) and S. palustre green part (76 OTUs) (Figure 3D).

The LEfSe analysis was conducted to identify indicator groups in different microhabitats (Figure 4). In S. palustre peat samples, 17 indicator species were identified which belonged to the phyla Acidobacteria, Bacteroidetes, Chloroflexi, and Nitrospirae, classes Acidobacteria, Dehalococcoidia, Nitrospira, Betaproteobacteria and Deltaproteobacteria. The indicator genus was Candidatus Solibacter in peat samples. In S. palustre brown part samples, 12 indicator species were enriched which were affiliated with the phylum Actinobacteria, classes Actinobacteria and Gammaproteobacteria. Afipia, Serratia, and Pseudomonas were indicator genera in the $S$. palustre brown part. In $S$. palustre green part samples, six indicator groups were enriched which fell into the phylum Cyanobacteria, class Cyanobacteria, order Rhodospirillales, family Acetobacteraceae, and genera Edaphobacter and Acidiphilium.

\section{Correlations Between Physicochemical Characteristics and S. palustre Peat Bacterial Communities}

Alpha diversity (OTU richness, ACE and Shannon indices) showed a significant correlation with water table as indicated by regression models $(p<0.05)$ (Figures 5A-C). Water table and total nitrogen were found to significantly affect microbial communities in $S$. palustre peat samples as indicated by RDA analysis (Figure 5D), which explained $51.3 \%$ and $22.1 \%$ of the variation, respectively. Bacterial communities from the NNF1 were positively correlated with total nitrogen and negatively correlated with water table, whereas those from the sixth site of Yangluchang (YLC6) were positively correlated with water table (Figure 5D). Specifically, Bacteroidetes $(r=0.922, p<0.01)$ and Chloroflexi $(r=0.683, p<0.05)$ were positively correlated with water table, whereas Acidobacteria $(r=-0.751, p<0.01)$ and Actinobacteria $(r=-0.751, p<0.01)$ were negatively correlated with water table (Supplementary Table S4). Bacteroidetes $(r=-0.613, p<0.05)$ were negatively correlated with total nitrogen (Supplementary Table S4). Briefly, the structure of bacterial communities in $S$. palustre peat altered from low water table NNF1 $(-8 \mathrm{~cm})$ to high water table YLC6 $(0 \mathrm{~cm})$ (Supplementary Figure S1 and Supplementary Table S5).

\section{DISCUSSION}

\section{Variation of Bacterial Microbiome Among Microhabitats}

Generally, bacterial communities are mainly composed of several dominant phyla in a wide range of soil types as well as in peatlands, e.g., Proteobacteria, Acidobacteria, Bacteroidetes, Actinobacteria, and Firmicutes (Griffiths et al., 2016; Karimi et al., 2018; Shi et al., 2018). Our results with Proteobacteria and Acidobacteria as the dominant peat microbial taxa were consistent with those in other peatlands (Kraigher et al., 2006; Ausec et al., 2009; Danilova et al., 2016).

Alpha diversity changed across microhabitats in our study. For example, a remarkable loss of alpha diversity (e.g., Shannon index from 7.02 in SB to 4.78 in SG) and decrease in total and specific OTUs (from 2,340 in SB to 1,633 in SG, and 
202 in SB to 76 in SG, respectively) (Figures 3A,D) were observed from $S$. palustre brown part to $S$. palustre green part which clearly showed a microhabitat differentiation. This differentiation was also confirmed by the clustering of community structure based on PCoA (Figure 3B).

These changes may closely relate to the specific cell types in green part and brown part of Sphagnum. Sphagnum green part consists of live photosynthetic cells (chlorocytes) and Sphagnum brown part composes of dead and water-filled hyaline cells (hyalocytes). Hyalocytes allow free movement of solutes or microorganisms in and out (Raghoebarsing et al., 2005; Shcherbakov et al., 2013), and further resulted in higher biodiversity of microbial communities in Sphagnum brown part. In contrast, microbial colonization in Sphagnum green part is strictly selected by chlorocytes (Opelt et al., 2007b; Bragina et al., 2012b), thus decreased microbial community diversity.

Moreover, the difference in the trophic status may also lead to the changes of bacterial microbiome in different microhabitats. The ratio of Proteobacteria to Acidobacteria has been used as an indicator of nutrient status in soil ecosystems (Smit et al., 2001) as well as in different peatlands (Hartman et al., 2008; Urbanová and Bárta, 2014). As a cosmopolitan taxon in acidic environments, Acidobacteria is able to grow under oligotrophic conditions (Philippot et al., 2010; Dedysh, 2011; Andersen et al., 2013). Proteobacteria are usually associated with higher carbon availability according to the copiotroph-oligotroph theory (Fierer et al., 2007; Leff et al., 2015). Thus, a higher ratio of Proteobacteria to Acidobacteria would indicate nutrient-rich conditions, or vice versa. Generally, species richness and microbial diversity increase with the improvement of nutritional status in peat sediments (Urbanová and Bárta, 2014). In our study, the ratio of Proteobacteria to Acidobacteria was $1.52 \pm 0.38$, $3.02 \pm 1.03$, and $7.08 \pm 1.78$ in $S$. palustre peat, S. palustre brown part, and $S$. palustre green part, respectively, indicating a relative nutrient-poor condition in Sphagnum peat compared with those in Sphagnum mosses. However, bacterial diversity decreased from $S$. palustre peat to $S$. palustre green part. This discrepancy was also observed previously (Xiang et al., 2014), which may result from the strict selection to microbiomes by mosses.

Besides variations in microbial diversity across microhabitats, indicator taxa also varied (Figure 4), which might indicate the microbial preference to specific microhabitats and the variation in microbial potential functions. Candidatus Solibacter affiliated with Acidobacteria are the indicator taxa in S. palustre peat, which were reported to be capable of utilizing various carbon sources, and reducing nitrate and nitrite under oligotrophic conditions (Ward et al., 2009) and further adapt to the acidic peatlands (Kanokratana et al., 2011). In this study, all the peat samples were acidic ( $\mathrm{pH}$ : 5.35-6.70) with a low content of total nitrogen (1.03-2.17\%), which may favor the growth of Candidatus Solibacter and thus result in their dominance. Serratia and Pseudomonas belonging to Gammaproteobacteria are the indicator groups in S. palustre brown part. Serratia were reported to be typical dwellers of Sphagnum plants in peat bogs (Opelt and Berg, 2004; Belova et al., 2006) and the major antagonists in S. fallax (Opelt et al., 2007c). Moreover, the isolated endophytic bacteria with high antagonistic potential were also confirmed to be Serratia and Pseudomonas in Sphagnum mosses (Shcherbakov et al., 2013). Therefore the dominance of Serratia and Pseudomonas in S. palustre brown part may associate the antagonistic potential of Sphagnum. Acidiphilium and Edaphobacter are the indicator species in S. palustre green part. Acidiphilium affiliated with Alphaproteobacteria are obligate acidophilic chemoorganotrophic bacteria (Okamura et al., 2015) and produce zincbacteriochlorophyll (Zn-BChl) a only under aerobic conditions (Hiraishi and Shimada, 2001). Compared with Mg-BChl a, $\mathrm{Zn}-\mathrm{BChl} \mathrm{a}$ is more stable and has advantages in photosynthesis under acidic environments. Edaphobacter are obligate aerobic and heterotrophic organisms, which may prefer neutral to slightly acidic environments and be isolated from a co-culture with a methanotrophic bacterium (Koch et al., 2008). Hence, the enriched Acidiphilium and Edaphobacter in S. palustre green part may benefit the growth of Sphagnum.

\section{Water Table and Total Nitrogen Shape Bacterial Communities in S. palustre Peat}

Multiple environmental factors have been demonstrated to affect peat microbial communities, e.g., water table (Kwon et al., 2013; Mishra et al., 2014; Chambers et al., 2016; Urbanová and Barta, 2016; Zhong et al., 2017), pH (Hartman et al., 2008; Lin et al., 2012), temperature (Pankratov et al., 2011), organic matter (Elliott et al., 2015), and $\mathrm{C} / \mathrm{P}$ ratio (Lin et al., 2014).

Our results showed that the decrease of water table altered alpha diversity of bacterial communities (OTU richness, ACE and Shannon indices) in $S$. palustre peat. Alpha diversity significantly (regression models, $p<0.05$ ) correlated with water table (Figures 5A-C). These changes of community diversity were probably related to the differences in peat physicochemical properties (Lauber et al., 2009; Zhong et al., 2017). In our study, the decrease of TOC and increase of TN content with water table decrease supported the perspective (Supplementary Table S1) and matched with observations reported from other peatlands (Urbanová and Bárta, 2014; Urbanová and Barta, 2016). Interestingly, the alpha diversity presented a unimodal correlation with water table change and higher value was observed at the oxic-anoxic interfaces $(-5 \mathrm{~cm})$. Microbial activities and diversity could be enhanced at the oxic-anoxic interface, in which the alteration of electron donors and acceptors often happens with the fluctuation of water table (Brune et al., 2000; Daffonchio et al., 2006). Besides the changes in alpha diversity, water table also significantly altered the composition and structure of bacterial communities (Supplementary Figure S1). The decrease in water table could influence community structure via several ways: altering the oxic-anoxic interface, increasing peat decomposability, and the bulk density of surface peat (Jaatinen et al., 2007; Urbanová and Barta, 2016). In our study, with the decrease of water table from YLC6 $(0 \mathrm{~cm})$ to EHB1 $(-5 \mathrm{~cm})$ or YLC2 $(-5 \mathrm{~cm})$ to NNF1 $(-8 \mathrm{~cm})$, more oxygen could penetrate into Sphagnum peat and increase the thickness of aerobic layer, which leads to the increase of the relative abundance of Actinobacteria, Alphaproteobacteria, and 


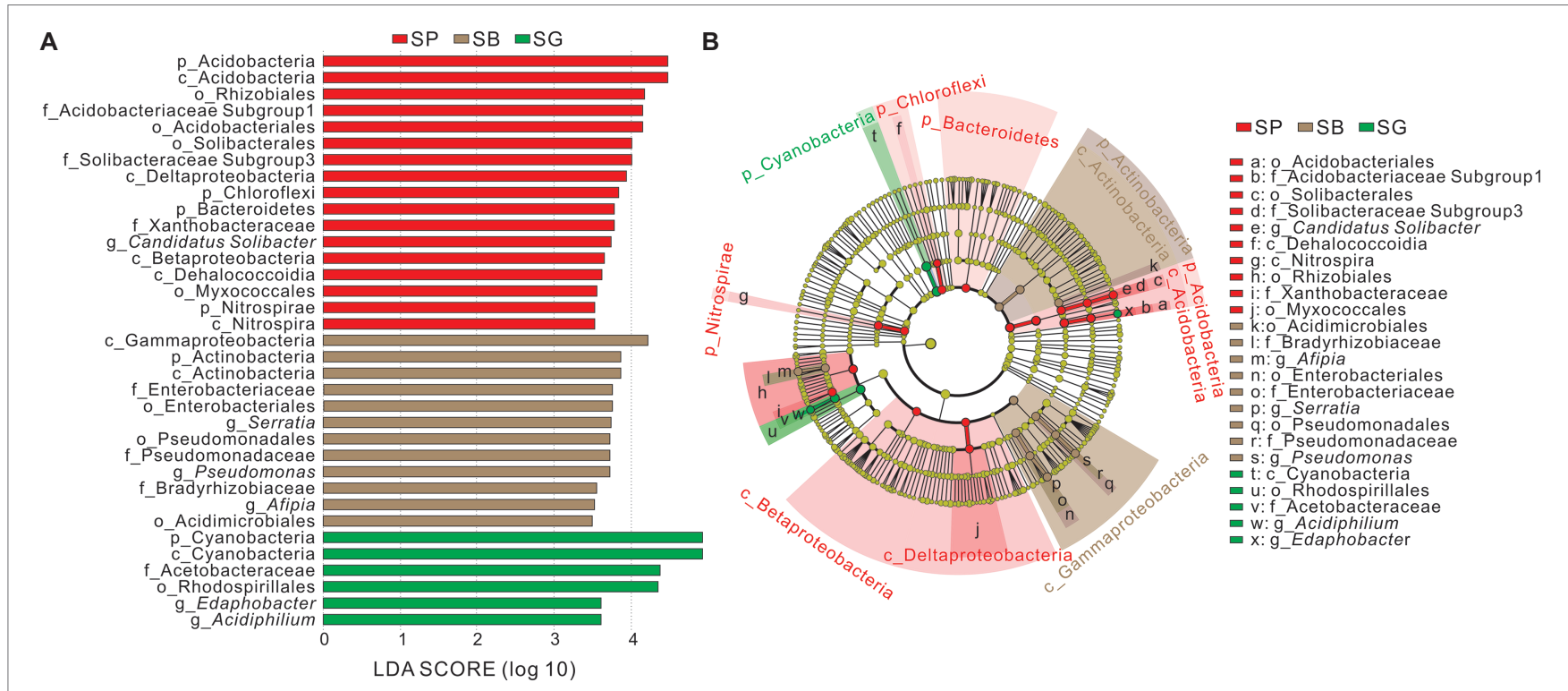

FIGURE 4 | Indicator groups analysis of bacterial communities in different microhabitats of S. palustre with LDA SCORE > 3.5 (A) and taxonomic cladogram (B) through linear discriminant analysis effect size (LEfSe). Nodes from inside to outside represent the phylogenetic levels from phylum to genus, respectively. Yellow nodes represent taxa that do not significantly discriminate among microhabitats. Significant discriminant taxa of S. palustre peat, S. palustre brown part, and S. palustre green part are highlighted in red, brown, and green, separately. The dimension of nodes is positively correlated with the relative abundance of taxon. Abbreviations are described in Figure 3.
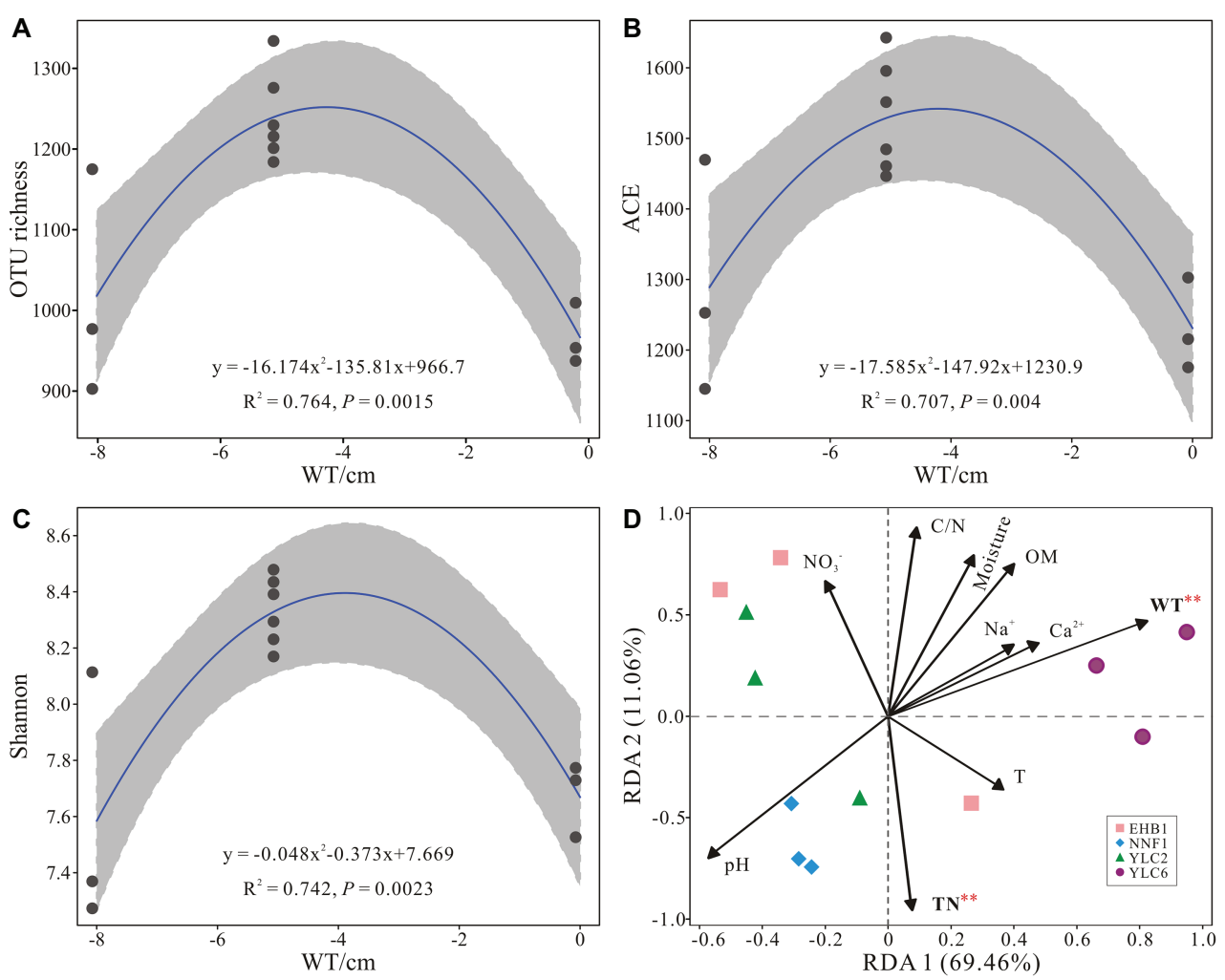

FIGURE 5 | Relationship between water table and alpha diversity of bacterial communities from S. palustre peat samples (A-C) and redundancy analysis showing the relationships between environmental factors and bacterial communities from $S$. palustre peat samples (D). Significant levels ( $p<0.01)$ are marked with red asterisk based on permutation test $(n=1,000)$. Abbreviations of environmental factors and sampling site are the same as those in Figure 2. 
Gammaproteobacteria, and decrease of the relative abundance of Deltaproteobacteria and Chloroflexi (Supplementary Table S5). Previous studies also show that with the decrease of water table, the relative abundance of Alphaproteobacteria and Gammaproteobacteria increases in a tropical peatland (Mishra et al., 2014), whereas Deltaproteobacteria decrease in bogs and fens (Urbanová and Barta, 2016). These results are consistent with ours in the Dajiuhu Peatland. Furthermore, the variation of water table affects microbial functional groups. For example, heterotrophic Actinobacteria can degrade recalcitrant polymeric substances such as lignin, chitin, pectin, aromatics, and humic acids (Killham and Prosser, 2015) under aerobic conditions and thus thrive in oxic layers in acidic peatlands (Jaatinen et al., 2007). Lower water table also significantly increased the relative abundance of aerobic Methylocystaceae (methanotrophs) (Supplementary Table S5), which may further impact $\mathrm{CH}_{4}$ emission in peatland ecosystems (Kwon et al., 2013; Zhong et al., 2017). In contrast, anaerobes such as Chloroflexi favor a higher water table.

Total nitrogen also significantly affected peat bacterial communities. Nitrogen is usually limited in boreal peatlands, especially Sphagnum-dominated peatlands (Wu and Blodau, 2013; Vile et al., 2014). Sphagnum mosses can obtain nitrogen via their symbiotic microbes, e.g., Bradyrhizobium, Beijerinckia, Burkholderia, Pseudomonas, Sphingobacterium, and methanotrophs (Methylobacterium and Methylocella) as well as Cyanobacteria (Bragina et al., 2012a, 2013). After death, Sphagnum mosses can serve as important $\mathrm{N}$-sources in peatland ecosystems, which can be converted to different $\mathrm{N}$ forms, e.g., $\mathrm{NH}_{4}{ }^{+}$(van den Elzen et al., 2017) under aerobic conditions, thus increasing the bioavailability of nitrogen (Rütting et al., 2011). In contrast, anaerobic conditions resulting from higher water table would lead to the rapid immobilization of nitrogen, thus decreasing microbial activity (Zhang et al., 2018).

\section{CONCLUSION}

In the present study, we confirmed that bacterial microbiome in the Dajiuhu Peatland, a subtropical peatland, shared the dominant bacterial phyla such as Proteobacteria and Acidobacteria with other peatlands. The microhabitats were found to play a fundamental role in the structure of bacterial

\section{REFERENCES}

Andersen, R., Chapman, S. J., and Artz, R. R. E. (2013). Microbial communities in natural and disturbed peatlands: a review. Soil Biol. Biochem. 57, 979-994. doi: $10.1016 /$ j.soilbio.2012.10.003

Ausec, L., Kraigher, B., and Mandic-Mulec, I. (2009). Differences in the activity and bacterial community structure of drained grasslandand forest peat soils. Soil Biol. Biochem. 41, 1874-1881. doi: 10.1016/j.soilbio.2009.06.010

Bahram, M., Hildebrand, F., Forslund, S., Anderson, J., Soudzilovskaia, N. A., Bodegom, P., et al. (2018). Structure and function of the global topsoil microbiome. Nature 560, 233-237. doi: 10.1038/s41586-018-0386-6

Belova, S. E., Pankratov, T. A., and Dedysh, S. N. (2006). Bacteria of the genus Burkholderia as a typical component of the microbial community of Sphagnum peat bogs. Microbiology 75, 110-117. doi: 10.1134/s0026261706010164 microbiome from $S$. palustre peat to $S$. palustre brown part and further to $S$. palustre green part. Community richness and alpha diversity were the highest in $S$. palustre peat and the lowest in $S$. palustre green part. Distinctive indicator groups identified in each microhabitat also supported our hypothesis about microhabitat differentiation driving the variations of bacterial microbiome. Cell types in different parts of Sphagnum may account for the difference in their bacterial microbiome. Water table and total nitrogen shaped the bacterial communities in $S$. palustre peat. These results enhance our understanding about microbial variation among microhabitats in subalpine peatland ecosystems. However, microbial function variations across different microhabitats needed to be investigated in near future.

\section{DATA AVAILABILITY}

The datasets generated for this study can be found in National Center for Biotechnology Information (NCBI), PRJNA512496.

\section{AUTHOR CONTRIBUTIONS}

WT conducted the experiments, analyzed data, and drafted the manuscript. HW designed the study, provided the funding, and revised the manuscript. XX helped with data analyses. RW and YX helped with sample collection.

\section{FUNDING}

This work was supported by the National Natural Science Foundation of China (41572325) and the Fundamental Research Funds for the Central Universities, China University of Geosciences (Wuhan), CUGCJ1703 and CUGQY1922.

\section{SUPPLEMENTARY MATERIAL}

The Supplementary Material for this article can be found online at: https://www.frontiersin.org/articles/10.3389/fmicb.2019.01661/ full\#supplementary-material

Berg, A., Danielsson, A., and Svensson, B. H. (2013). Transfer of fixed-N from $\mathrm{N}_{2}$-fixing Cyanobacteria associated with the moss Sphagnum riparium results in enhanced growth of the moss. Plant Soil 362, 271-278. doi: 10.1007/ s11104-012-1278-4

Braak, C. J. F. T., and Šmilauer, P. (2015). Topics in constrained and unconstrained ordination. Plant Ecol. 216, 1-14. doi: 10.1007/s11258-014-0356-5

Bragina, A., Berg, C., and Berg, G. (2015). The core microbiome bonds the Alpine bog vegetation to a transkingdom metacommunity. Mol. Ecol. 24, 4795-4807. doi: 10.1111/mec.13342

Bragina, A., Berg, C., Cardinale, M., Shcherbakov, A., Chebotar, V., and Berg, G. (2012b). Sphagnum mosses harbour highly specific bacterial diversity during their whole lifecycle. ISME J. 6, 802-813. doi: 10.1038/ismej.2011.151

Bragina, A., Berg, C., Müller, H., Moser, D., and Berg, G. (2013). Insights into functional bacterial diversity and its effects on Alpine bog ecosystem functioning. Sci. Rep. 3, 1955-1962. doi: 10.1038/srep01955 
Bragina, A., Oberauner-Wappis, L., Zachow, C., Halwachs, B., Thallinger, G. G., Müller, H., et al. (2014). The Sphagnum microbiome supports bog ecosystem functioning under extreme conditions. Mol. Ecol. 23, 4498-4510. doi: 10.1111/ mec. 12885

Bragina, A., Stefanie, M., Christian, B., Henry, M., Vladimir, C., Franz, H., et al. (2012a). Similar diversity of alphaproteobacteria and nitrogenase gene amplicons on two related Sphagnum mosses. Front. Microbiol. 2, 275-284. doi: $10.3389 /$ fmicb. 2011.00275

Brune, A., Frenzel, P., and Cypionka, H. (2000). Life at the oxic-anoxic interface: microbial activities and adaptations. FEMS Microbiol. Rev. 24, 691-710. doi: 10.1111/j.1574-6976.2000.tb00567.x

Cai, M., Hu, C., Wang, X., Zhao, Y., Jia, W., Sun, X., et al. (2019). Selenium induces changes of rhizosphere bacterial characteristics and enzyme activities affecting chromium/selenium uptake by pak choi (Brassica campestris L. ssp. Chinenis Makino) in chromium contaminated soil. Environ. Pollut. 249, 716-727. doi: 10.1016/j.envpol.2019.03.079

Caporaso, J. G., Kuczynski, J., Stombaugh, J., Bittinger, K., Bushman, F. D., Costello, E. K., et al. (2010). QIIME allows analysis of high-throughput community sequencing data. Nat. Methods 7, 335-336. doi: 10.1038/nmeth.f.303

Chambers, L. G., Guevara, R., Boyer, J. N., Troxler, T. G., and Davis, S. E. (2016). Effects of salinity and inundation on microbial community structure and function in a mangrove peat soil. Wetlands 36, 361-371. doi: 10.1007/ s13157-016-0745-8

Cole, J. R., Wang, Q., Cardenas, E., Fish, J., Chai, B., Farris, R. J., et al. (2009). The ribosomal database project: improved alignments and new tools for rRNA analysis. Nucleic Acids Res. 37, D141-D145. doi: 10.1093/nar/gkn879

Daffonchio, D., Borin, S., Brusa, T., Brusetti, L., van der Wielen, P. W. J. J., Bolhuis, H., et al. (2006). Stratified prokaryote network in the oxic-anoxic transition of a deep-sea halocline. Nature 440, 203-207. doi: 10.1038/ nature 04418

Danilova, O. V., Belova, S. E., Gagarinova, I. V., and Dedysh, S. N. (2016). Microbial community composition and methanotroph diversity of a subarctic wetland in Russia. Microbiology 85, 545-554. doi: 10.1134/S0026261716050039

Dedysh, S. N. (2011). Cultivating uncultured bacteria from northern wetlands: knowledge gained and remaining gaps. Front. Microbiol. 2, 184-198. doi: 10.3389/fmicb.2011.00184

Edgar, R. C. (2010). Search and clustering orders of magnitude faster than BLAST. Bioinformatics 26, 2460-2461. doi: 10.1093/bioinformatics/btq461

Edgar, R. C., Haas, B. J., Clemente, J. C., Quince, C., and Knight, R. (2011). UCHIME improves sensitivity and speed of chimera detection. Bioinformatics 27, 2194-2200. doi: 10.1093/bioinformatics/btr381

Elliott, D. R., Caporn, S. J. M., Nwaishi, F., Nilsson, R. H., and Sen, R. (2015). Bacterial and fungal communities in a degraded ombrotrophic peatland undergoing natural and managed re-vegetation. PLoS One 10, e0124726-e0124735. doi: 10.1371/journal.pone.0124726

Fierer, N., Bradford, M. A., and Jackson, R. B. (2007). Toward an ecological classification of soil bacteria. Ecology 88, 1354-1364. doi: 10.1890/05-1839

Gilbert, D., and Mitchell, E. A. D. (2006). "Microbial diversity in Sphagnum peatlands" in Peatlands: Evolution and records of environmental and climate changes. eds. I. P. Martini, A. M. Cortizas and W. Chesworth (Amsterdam: Elsevier), 287-318.

Griffiths, R. I., Thomson, B. C., Plassart, P., Gweon, H. S., Stone, D., Creamer, R. E., et al. (2016). Mapping and validating predictions of soil bacterial biodiversity using European and national scale datasets. Appl. Soil Ecol. 97, 61-68. doi: 10.1016/j.apsoil.2015.06.018

Hartman, W. H., Richardson, C. J., Vilgalys, R., and Bruland, G. L. (2008). Environmental and anthropogenic controls over bacterial communities in wetland soils. Proc. Natl. Acad. Sci. USA 105, 17842-17847. doi: 10.1073/ pnas.0808254105

Hiraishi, A., and Shimada, K. (2001). Aerobic anoxygenic photosynthetic bacteria with zinc-bacteriochlorophyll. J. Gen. Appl. Microbiol. 47, 161-180. doi: 10.2323/jgam.47.161

Horwáth, W. R., and Kessel, C. V. (2001). Acid fumigation of soils to remove carbonates prior to total carbon or carbon-13 isotopic analysis. Soil Sci. Soc. Am. J. 65, 1853-1856. doi: 10.2136/sssaj2001.1853

Huang, X., Meyers, P. A., Yu, J., Wang, X., Huang, J., Jin, F., et al. (2012). Moisture conditions during the Younger Dryas and the early Holocene in the middle reaches of the Yangtze River, central China. The Holocene 22, 1473-1479. doi: 10.1177/0959683612450202
Huang, X., Xue, J., Wang, X., Meyers, P. A., Huang, J., and Xie, S. (2013). Paleoclimate influence on early diagenesis of plant triterpenes in the Dajiuhu peatland, central China. Geochim. Cosmochim. Acta 123, 106-119. doi: 10.1016/j.gca.2013.09.017

Jaatinen, K., Fritze, H., Laine, J., and Laiho, R. (2007). Effects of short- and long-term water-level drawdown on the populations and activity of aerobic decomposers in a boreal peatland. Glob. Chang. Biol. 13, 491-510. doi: 10.1111/j.1365-2486.2006.01312.x

Jackson, C. R., Kong, C. L., and Yule, C. M. (2009). Structural and functional changes with depth in microbial communities in a tropical Malaysian peat swamp forest. Microb. Ecol. 57, 402-412. doi: 10.1007/s00248008-9409-4

Jassey, V. E., Gilbert, D., Binet, P., Toussaint, M. L., and Chiapusio, G. (2011). Effect of a temperature gradient on Sphagnum fallax and its associated living microbial communities: a study under controlled conditions. Can. J. Microbiol. 57, 226-235. doi: 10.1139/W10-116

Kanokratana, P., Uengwetwanit, T., Rattanachomsri, U., Bunterngsook, B., Nimchua, T., Tangphatsornruang, S., et al. (2011). Insights into the phylogeny and metabolic potential of a primary tropical peat swamp forest microbial community by metagenomic analysis. Microb. Ecol. 61, 518-528. doi: 10.1007/ s00248-010-9766-7

Karimi, B., Terrat, S., Dequiedt, S., Saby, N., Horrigue, W., Lelièvre, M., et al. (2018). Biogeography of soil bacteria and archaea across France. Sci. Adv. 4, eaat1808-eaat1821. doi: 10.1126/sciadv.aat 1808

Killham, K., and Prosser, J. I. (2015). "The bacteria and Archaea" in Soil microbiology, ecology and biochemistry. 4th Edn. ed. E. A. Paul (Boston: Academic Press), 41-76.

Kip, N., Fritz, C., Langelaan, E. S., and Pan, Y. (2012). Methanotrophic activity and diversity in different Sphagnum magellanicum dominated habitats in the southernmost peat bogs of Patagonia. Biogeosciences 9, 47-55. doi: 10.5194/bg-9-47-2012

Kip, N., Winden, J. F. V., Pan, Y., Bodrossy, L., Reichart, G. J., Smolders, A. J. P., et al. (2010). Global prevalence of methane oxidation by symbiotic bacteria in peat-moss ecosystems. Nat. Geosci. 3, 617-621. doi: 10.1038/ ngeo939

Koch, I. H., Frederic, G., Dunfield, P. F., and Overmann, J. (2008). Edaphobacter modestus gen. nov., sp. nov., and Edaphobacter aggregans sp. nov., acidobacteria isolated from alpine and forest soils. Int. J. Syst. Evol. Microbiol. 58, 1114-1122. doi: 10.1099/ijs.0.65303-0

Kostka, J. E., Weston, D. J., Glass, J. B., Lilleskov, E. A., Shaw, A. J., and Turetsky, M. R. (2016). The Sphagnum microbiome: new insights from an ancient plant lineage. New Phytol. 211, 57-64. doi: 10.1111/nph.13993

Kraigher, B., Stres, B., Hacin, J., Ausec, L., Mahne, I., Jdvan, E., et al. (2006). Microbial activity and community structure in two drained fen soils in the Ljubljana Marsh. Soil Biol. Biochem. 38, 2762-2771. doi: 10.1016/j. soilbio.2006.04.031

Kulichevskaia, I. S., Pankratov, T. A., and Dedysh, S. N. (2006). Detection of representatives of the Planctomycetes in Sphagnum peat bogs by molecular and cultivation methods. Microbiology 75, 329-335. doi: 10.1134/ S0026261706030155

Kwon, M. J., Haraguchi, A., and Kang, H. (2013). Long-term water regime differentiates changes in decomposition and microbial properties in tropical peat soils exposed to the short-term drought. Soil Biol. Biochem. 60, 33-44. doi: 10.1016/j.soilbio.2013.01.023

Lauber, C. L., Hamady, M., Knight, R., and Fierer, N. (2009). Pyrosequencingbased assessment of soil $\mathrm{pH}$ as a predictor of soil bacterial community structure at the continental scale. Appl. Environ. Microbiol. 75, 5111-5120. doi: 10.1128/AEM.00335-09

Leff, J. W., Jones, S. E., Prober, S. M., Barberán, A., Borer, E. T., Firn, J. L., et al. (2015). Consistent responses of soil microbial communities to elevated nutrient inputs in grasslands across the globe. Proc. Natl. Acad. Sci. USA 112, 10967-10972. doi: 10.1073/pnas.1508382112

Li, Y., Ma, C., Zhu, C., Huang, R., and Zheng, C. (2016). Historical anthropogenic contributions to mercury accumulation recorded by a peat core from Dajiuhu montane mire, central China. Environ. Pollut. 216, 332-339. doi: 10.1016/j. envpol.2016.05.083

Lin, X., Green, S., Tfaily, M. M., Prakash, O., Konstantinidis, K. T., Corbett, J. E., et al. (2012). Microbial community structure and activity linked to contrasting biogeochemical gradients in bog and fen environments of the Glacial Lake 
Agassiz Peatland. Appl. Environ. Microbiol. 78, 7023-7031. doi: 10.1128/ AEM.01750-12

Lin, X., Tfaily, M. M., Steinweg, J. M., Chanton, P., and Esson, K. (2014). Microbial community stratification linked to utilization of carbohydrates and phosphorus limitation in a boreal peatland at Marcell Experimental Forest, Minnesota, USA. Appl. Environ. Microbiol. 80, 3518-3530. doi: 10.1128/ AEM.00205-14

Mishra, S., Lee, W. A., Hooijer, A., Reuben, S., Sudiana, I. M., Idris, A., et al. (2014). Microbial and metabolic profiling reveal strong influence of water table and land-use patterns on classification of degraded tropical peatlands. Biogeosciences 11, 14009-14042. doi: 10.5194/bg-11-1727-2014

Mitsch, W. J., Nahlik, A. M., Mander, Ü., Zhang, L., Anderson, C. J., Jørgensen, S. E., et al. (2013). Wetlands, carbon, and climate change. Landsc. Ecol. 28, 583-597. doi: $10.1007 /$ s10980-012-9758-8

Okamura, K., Kawai, A., Wakao, N., Yamada, T., and Hiraishi, A. (2015). Acidiphilium iwatense sp. nov., isolated from an acid mine drainage treatment plant, and emendation of the genus Acidiphilium. Int. J. Syst. Evol. Microbiol. 65, 42-48. doi: 10.1099/ijs.0.065052-0

Opelt, K., and Berg, G. (2004). Diversity and antagonistic potential of bacteria associated with bryophytes from nutrient-poor habitats of the Baltic Sea Coast. Appl. Environ. Microbiol. 70, 6569-6579. doi: 10.1128/AEM.70.11. 6569-6579.2004

Opelt, K., Berg, C., and Berg, G. (2007a). The bryophyte genus Sphagnum is a reservoir for powerful and extraordinary antagonists and potentially facultative human pathogens. FEMS Microbiol. Ecol. 61, 38-53. doi: 10.1111/j. 1574-6941.2007.00323.x

Opelt, K., Berg, C., Schönmann, S., Eberl, L., and Berg, G. (2007b). High specificity but contrasting biodiversity of Sphagnum-associated bacterial and plant communities in bog ecosystems independent of the geographical region. ISME J. 1, 502-516. doi: 10.1038/ismej.2007.58

Opelt, K., Chobot, V., Hadacek, F., Schönmann, S., Eberl, L., and Berg, G. $(2007 \mathrm{c})$. Investigations of the structure and function of bacterial communities associated with Sphagnum mosses. Environ. Microbiol. 9, 2795-2809. doi: $10.1111 / j .1462-2920.2007 .01391 . x$

Pankratov, T. A., Ivanova, A. O., Dedysh, S. N., and Werner, L. (2011). Bacterial populations and environmental factors controlling cellulose degradation in an acidic Sphagnum peat. Environ. Microbiol. 13, 1800-1814. doi: 10.1111/j. 1462-2920.2011.02491.x

Pankratov, T. A., Serkebaeva, Y. M., Kulichevskaya, I. S., Liesack, W., and Dedysh, S. N. (2008). Substrate-induced growth and isolation of Acidobacteria from acidic Sphagnum peat. ISME J. 2, 551-560. doi: 10.1038/ismej.2008.7

Philippot, L., Andersson, S. G., Battin, T. J., Prosser, J. I., Schimel, J. P., Whitman, W. B., et al. (2010). The ecological coherence of high bacterial taxonomic ranks. Nat. Rev. Microbiol. 8, 523-529. doi: 10.1038/nrmicro2367

Raghoebarsing, A. A., Smolders, A. J., Schmid, M. C., Rijpstra, W. I., Woltersarts, M., Derksen, J., et al. (2005). Methanotrophic symbionts provide carbon for photosynthesis in peat bogs. Nature 436, 1153-1156. doi: 10.1038/ nature 03802

Rütting, T., Boeckx, P., Müller, C., and Klemedtsson, L. (2011). Assessment of the importance of dissimilatory nitrate reduction to ammonium for the terrestrial nitrogen cycle. Biogeosciences 8, 1779-1791. doi: 10.5194/bg-8-1779-2011

Schloss, P. D., Westcott, S. L., Ryabin, T., Hall, J. R., Hartmann, M., Hollister, E. B., et al. (2009). Introducing mothur: open-source, platform-independent, community-supported software for describing and comparing microbial communities. Appl. Environ. Microbiol. 75, 7537-7541. doi: 10.1128/ AEM.01541-09

Segata, N., Izard, J., Waldron, L., Gevers, D., Miropolsky, L., Garrett, W. S., et al. (2011). Metagenomic biomarker discovery and explanation. Genome Biol. 12, R60-R77. doi: 10.1186/gb-2011-12-6-r60

Shaw, A. J., Cox, C. J., and Boles, S. B. (2010). Global patterns in peatmoss biodiversity. Mol. Ecol. 12, 2553-2570. doi: 10.1046/j.1365-294X.2003.01929.x

Shcherbakov, A. V., Bragina, A. V., Kuzmina, E. Y., Berg, C., Muntyan, A. N., Makarova, N. M., et al. (2013). Endophytic bacteria of Sphagnum mosses as promising objects of agricultural microbiology. Microbiology 82, 306-315. doi: $10.1134 / S 0026261713030107$

Shi, Y., Li, Y., Xiang, X., Sun, R., Yang, T., He, D., et al. (2018). Spatial scale affects the relative role of stochasticity versus determinism in soil bacterial communities in wheat fields across the North China Plain. Microbiome 6, 27-39. doi: 10.1186/s40168-018-0409-4
Smit, E., Leeflang, P., Gommans, S., van den Broek, J., van Mil, S., and Wernars, K. (2001). Diversity and seasonal fluctuations of the dominant members of the bacterial soil community in a wheat field as determined by cultivation and molecular methods. Appl. Environ. Microbiol. 67, 2284-2291. doi: 10.1128/AEM.67.5.2284-2291.2001

Soudzilovskaia, N. A., Cornelissen, J. H., During, H. J., van Logtestijn, R. S., Lang, S. I., and Aerts, R. (2010). Similar cation exchange capacities among bryophyte species refute a presumed mechanism of peatland acidification. Ecology 91, 2716-2726. doi: 10.1890/09-2095.1

Urbanová, Z., and Bárta, J. (2014). Microbial community composition and in silico predicted metabolic potential reflect biogeochemical gradients between distinct peatland types. FEMS Microbiol. Ecol. 90, 633-646. doi: 10.1111/ $1574-6941.12422$

Urbanová, Z., and Barta, J. (2016). Effects of long-term drainage on microbial community composition vary between peatland types. Soil Biol. Biochem. 92, 16-26. doi: 10.1016/j.soilbio.2015.09.017

van den Elzen, E., Ljl, V. D. B., Van, d. W. B., Fritz, C., Sheppard, L. J., and Lpm, L. (2017). Effects of airborne ammonium and nitrate pollution strongly differ in peat bogs, but symbiotic nitrogen fixation remains unaffected. Sci. Total Environ. 610-611, 732-740. doi: 10.1016/j.scitotenv.2017.08.102

Vile, M. A., Wieder, R. K., Živković, T., Scott, K. D., Vitt, D. H., Hartsock, J. A., et al. (2014). $\mathrm{N}_{2}$-fixation by methanotrophs sustains carbon and nitrogen accumulation in pristine peatlands. Biogeochemistry 121, 317-328. doi: 10.1007/ s10533-014-0019-6

Ward, N. L., Challacombe, J. F., Janssen, P. H., Bernard, H., Coutinho, P. M., Martin, W., et al. (2009). Three genomes from the phylum Acidobacteria provide insight into the lifestyles of these microorganisms in soils. Appl. Environ. Microbiol. 75, 2046-2056. doi: 10.1128/AEM.02294-08

Weston, D. J., Timm, C. M., Walker, A. P., Gu, L., Muchero, W., Schmutz, J., et al. (2015). Sphagnum physiology in the context of changing climate: emergent influences of genomics, modeling and host-microbiome interactions on understanding ecosystem function. Plant Cell Environ. 38, 1737-1751. doi: 10.1111 pce. 12458

Wu, Y., and Blodau, C. (2013). PEATBOG: a biogeochemical model for analyzing coupled carbon and nitrogen dynamics in northern peatlands. Geosci. Model Dev. 6, 1173-1207. doi: 10.5194/gmd-6-1173-2013

Xiang, X., Wang, H. M., Gong, L. F., and Liu, Q. (2014). Vertical variations and associated ecological function of bacterial communities from Sphagnum to underlying sediments in Dajiuhu Peatland. Sci. China Earth Sci. 57, 1013-1020. doi: 10.1007/s11430-013-4752-9

Xiang, X., Wang, R., Wang, H., Gong, L., Man, B., and Xu, Y. (2017). Distribution of Bathyarchaeota communities across different terrestrial settings and their potential ecological functions. Sci. Rep. 7, 45028-45038. doi: 10.1038/srep45028

Yu, Z., Loisel, J., Brosseau, D. P., Beilman, D. W., and Hunt, S. J. (2010). Global peatland dynamics since the last glacial maximum. Geophys. Res. Lett. 37, L13402-L13406. doi: 10.1029/2010GL043584

Yun, Y., Wang, H., Man, B., Xiang, X., Zhou, J., Qiu, X., et al. (2016). The relationship between $\mathrm{pH}$ and bacterial communities in a single karst ecosystem and its implication for soil acidification. Front. Microbiol. 7, 1955-1968. doi: $10.3389 /$ fmicb.2016.01955

Zhang, J., Jiao, S., and Lu, Y. (2018). Biogeographic distribution of bacterial, archaeal and methanogenic communities and their associations with methanogenic capacity in Chinese wetlands. Sci. Total Environ. 622-623, 664-675. doi: 10.1016/j.scitotenv.2017.11.279

Zhong, Q., Chen, H., Liu, L., He, Y., Zhu, D., Jiang, L., et al. (2017). Water table drawdown shapes the depth-dependent variations in prokaryotic diversity and structure in Zoige peatlands. FEMS Microbiol. Ecol. 93:fix049. doi: 10.1093/femsec/fix049

Conflict of Interest Statement: The authors declare that the research was conducted in the absence of any commercial or financial relationships that could be construed as a potential conflict of interest.

Copyright (c) 2019 Tian, Wang, Xiang, Wang and Xu. This is an open-access article distributed under the terms of the Creative Commons Attribution License (CC BY). The use, distribution or reproduction in other forums is permitted, provided the original author(s) and the copyright owner(s) are credited and that the original publication in this journal is cited, in accordance with accepted academic practice. No use, distribution or reproduction is permitted which does not comply with these terms. 